\title{
CENTRALISMO Y FORMAS FLUIDAS DE PLANIFICACIÓN TERRITORIAL EN CHILE \\ Mecanismos de gobernanza horizontal para la agenda urbana local ${ }^{1}$

\author{
Centralism and Fluid Forms of Urban Planning \\ Mechanisms for Horizontal Governance for Local Urban Agendas
}

\author{
Marshall, Catalina \\ Investigadora Postdoctoral CEDEUS \\ Académica del Instituto de Estudios Urbanos y Territoriales \\ Pontificia Universidad Católica de Chile \\ cmarshal@uc.cl
}

\section{RESUMEN}

En el contexto del alto nivel de centralismo del Estado chileno, el país ha experimentado un proceso tardío y fragmentado de la descentralización de la planificación territorial, en el sentido de un traspaso significativo de atribuciones, competencias y facultades a gobiernos regionales y municipalidades. Ello resulta complejo bajo la premisa de la importancia de la descentralización gubernamental para el desarrollo de los territorios (Tocqueville, 1835; Borja y Castells, 1998; Boisier, 2010; OECD, 2013). En este artículo, se abordarán las complejidades de gobernanza de temas de planificación territorial, entre el nivel central y el nivel local (municipalidades) en Chile, desde el grado de incidencia de los municipios, en la formulación, diseño e implementación de grandes proyectos de carácter urbano y de inversión sectorial. Se expondrán los resultados enfocándose en casos claves, para demostrar nuevas modalidades de planificación, gestión y gobernanza urbana, mediante la colaboración y coordinación entre los niveles de gobierno, el sector privado, el sector académico, y la ciudadanía.

Palabras clave: Chile, descentralización, planificación territorial, espacios fluidos de gobernanza Bloque temático: Análisis y proyecto territorial

\begin{abstract}
Given the high level of centralism of the Chilean State, the country has experienced a delayed and fragmented process of decentralization, in the sense of a significant transfer of planning powers to regional governments and municipalities. This is complex under the premise of the importance of government decentralization for the development of territories (Tocqueville, 1835, Borja and Castells, 1998, Boisier, 2010, OECD, 2013). This article addresses the complexities of governance of territorial planning issues between the central level and the local level (municipalities) in Chile, from the degree of incidence of municipalities, in the formulation, design and implementation of large urban and sectoral investment projects. Results will be presented focusing on key cases, to show new modalities of planning, management and urban governance, through collaboration and coordination between levels of government, the private sector, the academic sector, and citizens.
\end{abstract}

Keywords: Chile, decentralization, territorial planning, fluid governance spaces

Topic: Analysis and Territorial project

\footnotetext{
${ }^{1}$ Investigación realizada con financiamiento de la Comisión Nacional de Investigación Científica (CONICYT) - Programa de Beca de Doctorado Nacional
} 


\section{Introducción}

En relación con el intenso desarrollo urbano que se ha experimentado en Chile durante el s. XX y a la estructura de la gobernanza que ha surgido para administrarlo principalmente, se reconoce una institucionalidad de carácter centralizada y sectorializada, que ha tenido pocos cambios desde su consolidación (OECD, 2013). En ese contexto, resulta esperable que el país también haya experimentado un proceso tardío y fragmentado de la descentralización de la planificación territorial, en el sentido de un traspaso significativo de atribuciones, competencias y facultades de planificación a gobiernos regionales y municipalidades (Marshall, 2018). Más específicamente, desde la promulgación de la Constitución Política de 1925, ha prevalecido un modelo centralizado con alta injerencia sectorial en la aprobación de instrumentos de planificación, en la aplicación de las disposiciones de la Ley General de Urbanismo y Construcciones (LGUC), y en la gestión de grandes proyectos urbanos en áreas urbanas a lo largo del país (Ibid).

A raíz del cuestionamiento sobre el beneficio de descentralizar la institucionalidad e instrumentos de la planificación territorial, en este artículo se toma en consideración la discusión en torno a la importancia de la descentralización gubernamental y el rol de los gobiernos regionales y locales para el desarrollo de los territorios (Tocqueville, 1835; Borja y Castells, 1998; Boisier, 2010; OECD, 2013), a modo de premisa para analizar el caso chileno. También se considera la perspectiva de Rondinelli (1981), que plantea que la descentralización puede facilitar la articulación e implementación de las políticas de desarrollo diseñadas para lograr crecimiento con equidad, fortaleciendo la capacidad de las unidades regionales y subregionales, y capacitando a los líderes políticos a identificar sus propios problemas y prioridades de desarrollo. Más recientemente, también se ha desarrollado una literatura que discute los desafíos y oportunidades que enfrentan los gobiernos subnacionales en materias de gobernanza urbana y planificación territorial en un nuevo contexto de urbanización de escala regional y metropolitana (Brenner, 2003, 2004, 2006; Elinbaum y Galland, 2016).

Si se revisan los hitos legislativos que hicieron mención de temas de descentralización de la planificación territorial en Chile desde 1925 (Tabla 1), se puede ver que un primer caso es la Constitución Política de 1925, cuyo objetivo era reformar el sistema político, fijando un régimen representativo, presidencialista y con estricta separación de poderes; sus contenidos sí abordaron aspectos de gestión urbana a nivel municipal aunque sin delimitar bien el rol de los gobiernos locales y de los ministerios sectoriales, lo cual llevó a una disputa en la definición de los roles de cada cual. En 1931, el Decreto con Fuerza de Ley 345, cuyo objetivo era presentar las disposiciones relativas a la planificación urbana, urbanización y construcción en el territorio nacional, especifica recién en 1952, conceptos como metrópolis, intermunicipio, microrregión y región. En 1969, el Decreto Ley $\mathrm{N}^{\circ} 1.104$ oficializó un esquema regional que nunca fue implementado como política administrativa. El Decreto $N^{\circ} 1.104$ no abordó temas de gestión o planificación territorial, ni detalla atribuciones, competencias o facultades.

En 1974, los decretos supremos $\mathrm{N}^{\circ} 573$ y 575 tuvieron como objetivo implementar el proyecto de la regionalización de Chile, una iniciativa del régimen militar que tomó el poder del país en 1973. Específicamente en el Decreto $N^{\circ} 573$, además de establecer una nueva división geopolítica del país, se abordan temas de gestión urbana, planificación territorial tanto a nivel central, como a nivel regional y comunal. Un año después, se promulga el Decreto Supremo $N^{\circ} 458$, la Ley General de Urbanismo y Construcciones; su objetivo era presentar las disposiciones relativas a la planificación urbana, urbanización y construcción en el territorio nacional. Específicamente con respecto a la descentralización de la planificación urbana, en el artículo 27 se definió el concepto de la planificación urbana y en el artículo 28 se establecieron cuatro niveles de acción dentro de la planificación: nacional, regional, intercomunal y comunal. 


\begin{tabular}{|c|c|c|c|}
\hline $\begin{array}{l}\text { Documento } \\
\text { legislativo }\end{array}$ & Año & $\begin{array}{c}\text { Objetivo legislación | ¿Aborda } \\
\text { temas de planificación territorial o } \\
\text { gestión urbana? }\end{array}$ & $\begin{array}{l}\text { Atribuciones, competencias y } \\
\text { facultades descentralizadas }\end{array}$ \\
\hline $\begin{array}{l}\text { Constitución } \\
\text { Política }\end{array}$ & 1925 & $\begin{array}{l}\text { Reforma al sistema político, fijando } \\
\text { un régimen representativo, } \\
\text { presidencialista y con estricta } \\
\text { separación de poderes. } \\
\text { Sí aborda temas de gestión urbana a } \\
\text { nivel municipal. }\end{array}$ & $\begin{array}{l}\text { Establece que las municipalidades } \\
\text { deben cuidar de la construcción y } \\
\text { reparación de los caminos, calzadas, } \\
\text { puentes y de todas las obras de } \\
\text { necesidad, utilidad y ornato que se } \\
\text { costeen con fondos municipales. }\end{array}$ \\
\hline $\begin{array}{l}\text { Decreto con Fuerza } \\
\text { de Ley } N^{\circ} 345\end{array}$ & 1931 & $\begin{array}{l}\text { Su principal objetivo era presentar } \\
\text { las disposiciones relativas a la } \\
\text { planificación urbana, urbanización y } \\
\text { construcción en el territorio nacional. }\end{array}$ & $\begin{array}{l}\text { En } 1952 \text { se hicieron ajustes a la ley, } \\
\text { precisando por primera vez los } \\
\text { conceptos de metrópolis, } \\
\text { intermunicipio, microregión y región; } \\
\text { acción que refleja la necesidad de } \\
\text { acercarse a una nueva escala de } \\
\text { planificación (MINVU, 2015). }\end{array}$ \\
\hline $\begin{array}{l}\text { Regionalización de } \\
\text { Chile (Decreto 573) }\end{array}$ & 1974 & $\begin{array}{l}\text { Implementar el proyecto de } \\
\text { Regionalización de Chile. Aborda } \\
\text { temas de gestión urbana. Aborda } \\
\text { temas de planificación territorial a } \\
\text { nivel central, regional y comunal. }\end{array}$ & $\begin{array}{l}\text { I. Gobierno central: "Le } \\
\text { corresponderá definir las áreas } \\
\text { metropolitanas y establecer para } \\
\text { ellas régimenes especiales de } \\
\text { gobiernos y administración". } \\
\text { II. Nivel provincial: "Al gobernador le } \\
\text { corresponderá fiscalizar la ejecución } \\
\text { de los planes, programas y } \\
\text { proyectos de desarrollo de las } \\
\text { provincias". } \\
\text { III. Nivel comunal: "Estará a cargo de } \\
\text { la planificación y ejecución del } \\
\text { desarrollo económico y social de la } \\
\text { comuna o agrupación de comunas". }\end{array}$ \\
\hline $\begin{array}{l}\text { Regionalización de } \\
\text { Chile (Decreto 575) }\end{array}$ & 1974 & $\begin{array}{l}\text { Profundiza en el rol y facultades del } \\
\text { Intendente Regional, del Consejo } \\
\text { Regional de Desarrollo y de la } \\
\text { Secretaría Regional de Planificación } \\
\text { y Coordinación. Especifica el rol de } \\
\text { las Secretarías Regionales } \\
\text { Ministeriales (SEREMIs). No aborda } \\
\text { el tema de la planificación territorial } \\
\text { directamente. }\end{array}$ & $\begin{array}{l}\text { El decreto no define atribuciones, } \\
\text { competencias o facultades } \\
\text { específicas para niveles de gobierno, } \\
\text { sino que se enfoca en la definición } \\
\text { de órganos complementarios } \\
\text { sectoriales. }\end{array}$ \\
\hline $\begin{array}{l}\text { Decreto Supremo } \\
458 \text { (Ley General } \\
\text { de Urbanismo y } \\
\text { Construcciones) }\end{array}$ & 1975 & $\begin{array}{l}\text { Presentar las disposiciones relativas } \\
\text { a la planificación urbana, } \\
\text { urbanización y construcción en el } \\
\text { territorio nacional. }\end{array}$ & $\begin{array}{l}\text { Con respecto a la descentralización } \\
\text { de la planificación urbana, en el art. } \\
27 \text { se definió la planificación urbana } \\
\text { y en el art. } 28 \text { se establecieron } \\
\text { cuatro niveles de acción dentro de la }\end{array}$ \\
\hline
\end{tabular}




\begin{tabular}{|c|c|c|c|}
\hline & & & $\begin{array}{l}\text { planificación: nacional, regional, } \\
\text { intercomunal y comunal. }\end{array}$ \\
\hline $\begin{array}{l}\text { Ley } 19.130 \\
\text { (modifica la Ley } \\
\text { 18.695 LOCM) }\end{array}$ & 1992 & $\begin{array}{l}\text { Reestablecer y modernizar } \\
\text { definiciones de la Ley Orgánica } \\
\text { Constitucional de Municipalidades de } \\
\text { 1988. Sí aborda la planificación } \\
\text { territorial, pero solo a nivel municipal. }\end{array}$ & $\begin{array}{l}\text { I. Consejo de Desarrollo Comunal: } \\
\text { Debe encargarse de elaboración del } \\
\text { PRC y Pladeco. } \\
\text { II. Consejo Económico y Social } \\
\text { Comunal: Asegurar la participación } \\
\text { de organizaciones comunitarias en } \\
\text { los procesos de planificación. }\end{array}$ \\
\hline $\begin{array}{l}\text { Ley } 19.175 \\
\text { (LOCGAR) }\end{array}$ & 1993 & $\begin{array}{l}\text { Implementar y definir los Gobiernos } \\
\text { Regionales. Aborda la planificación } \\
\text { territorial a nivel regional. }\end{array}$ & $\begin{array}{l}\text { Consejo Regional. Deberá } \\
\text { establecer políticas y objetivos para } \\
\text { el desarrollo integral de los } \\
\text { asentamientos humanos de la } \\
\text { región. Deberá aprobar el PRC, PRIs } \\
\text { y opinar sobre los planes regionales. }\end{array}$ \\
\hline $\begin{array}{l}\text { Ley } 21.074 \text { de } \\
\text { Fortalecimiento de } \\
\text { la Regionalización }\end{array}$ & 2018 & $\begin{array}{l}\text { Profundizar la Regionalización. } \\
\text { Aborda la planificación territorial, } \\
\text { pero a nivel regional. }\end{array}$ & $\begin{array}{l}\text { I. Realizar el diseño, elaboración, } \\
\text { aprobación y aplicación de políticas, } \\
\text { planes, programas y proyectos. } \\
\text { II. Elaborar el PROT (carácter } \\
\text { vinculante). } \\
\text { III. Facultad regional para decidir } \\
\text { sobre localización de disposición de } \\
\text { residuos sólidos domiciliarios. }\end{array}$ \\
\hline
\end{tabular}

Tabla 1. Menciones a temas de planificación territorial en la legislación de descentralización

Fuente: Elaboración propia

En 1992, tras el retorno a la democracia en Chile, se promulga la Ley $N^{\circ} 19.130$ que modifica la Ley 18.695 (Ley Orgánica Constitucional de Municipalidades), con el objetivo de reestablecer y modernizar las definiciones de la versión anterior de la ley. La modificación legislativa sí abordó temas de planificación territorial, pero directamente a nivel municipal. En el nivel municipal, el Consejo de Desarrollo Comunal debería encargarse de la elaboración del Plan Regional Comunal (PRC), y de elaborar, aprobar y modificar el Plan de Desarrollo Comunal. A su vez, el Consejo Económico y Social Comunal, debería encargarse de asegurar la participación de organizaciones comunitarias en los procesos de planificación. En 1993, se promulga la Ley № 19.175 (Ley Orgánica Constitucional de Gobierno y Administración Regional), con el objetivo de implementar y definir los Gobiernos Regionales en Chile. En esta ley se aborda la planificación territorial, pero solo a nivel regional. Por ejemplo, se detalla que el Consejo Regional deberá establecer políticas y objetivos para el desarrollo integral de los asentamientos humanos de la región, y que deberá aprobar el Plan Regulador Comunal (PRC), planes intercomunales y opinar sobre planes regionales.

A su vez, la recientemente promulgada Ley №21.074 de Fortalecimiento de la Regionalización (2018), tuvo como objetivo profundizar algunos aspectos de la regionalización, en particular a lo que se refiere al establecimiento de nuevas competencias para los gobiernos regionales (GORE) en materias de ordenamiento y planificación territorial (permite la definición de áreas metropolitanas para áreas urbanas que cumplan con ciertos requisitos). Esta ley constituye un hito importante ya que por primera vez se hace un esfuerzo por concretar un real traspaso de competencias a los GORE, otorgándoles responsabilidades, por ejemplo, en la 
aprobación de instrumentos de planificación territorial. La promulgación de esta ley representa un proceso que comenzó a concretarse el año 2013 con la promulgación de la Ley № 20.678, la cual estableció la elección de los consejeros regionales; luego con la Ley № 20.990 del año 2017 se oficializó la elección directa de los gobernadores regionales para liderar el órgano ejecutivo de los GORE y presidir los consejos regionales (CORE); en tercer lugar se promulgó la Ley № 21.073 del año 2017 que regula la elección de gobernadores regionales. Durante el año 2019, se publicará el reglamento que permitirá su entrada en vigor (Ley № 21.074, 2018).

De esta manera, se puede observar que históricamente sí se ha abordado incrementalmente la descentralización, sin embargo, la legislación que definió una mayor cantidad de atribuciones, competencias y facultades para los gobiernos regionales y municipales, se concentró a fines del s. XX². A su vez, dichos procesos de descentralización mencionados mantuvieron un rol de injerencia desde el nivel central, con excepción de la Ley $N^{\circ} 21.074$ del 2018. En base a estas discusiones, se abordarán las complejidades de gobernanza ${ }^{3}$ de temas de planificación territorial, entre el nivel central y el nivel local (municipalidades) en Chile, desde la perspectiva del grado de incidencia de los municipios en la planificación conducida desde el nivel central (específicamente en la formulación, diseño e implementación de grandes proyectos de carácter urbano y de inversión sectorial). Son dos los objetivos de la investigación: (1) analizar cómo y en qué medida los municipios logran incidir en decisiones con respecto a proyectos de carácter urbano, financiados sectorialmente desde el nivel central; y (2) extraer lecciones y experiencias a partir de los casos de municipios que han logrado un mayor diálogo-con respecto a proyectos de carácter urbano-con el gobierno central. A modo de hipótesis, se plantea que la planificación urbana centralizada (expresada para este caso en proyectos urbanos de gran escala financiados sectorialmente), incide de manera disímil en la planificación territorial local y en la agenda urbana de proyectos, según características de comunas.

\section{Marco teórico}

El concepto de descentralización gubernamental ha sido ampliamente definido y clasificado según grados y tipos (Rondinelli et al., 1984 y 1989; Palma y Rufián, 1989; Boisier, 2010; Triesman, 2007; Falleti; 2010). La teoría de la descentralización como método para la organización de la democracia se atribuye a una corriente que se fundamenta en el poder local y la práctica del autogobierno, la cual fue influyente sobre todo en países anglosajones durante el siglo XIX; la primera persona en vincular el potencial del concepto de descentralización con gobernanza y local fue Alexis de Tocqueville, y sus ideas se vieron reflejadas en su obra Democracia en América (1835) (Boisier, 2010). Tocqueville proponía que el principio de la soberanía del Estado estaba presente en cada individuo de una sociedad (Finot, 2001). En América Latina, los procesos de descentralización gubernamental se vieron influidos y gatillados por las ideas de Tocqueville, cuando su libro llegó traducido a la ciudad de Buenos Aires (Nickson, 2003).

Definir y delimitar el concepto de planificación territorial, resulta igualmente desafiante; para Berke, Godschalk \& Kaiser (2006), se refiere a la definición de valores para organizar una serie de sistemas (económicos, medioambientales, uso de suelo, transporte, infraestructura), mediante una red de planes y políticas de diferentes escalas, proceso que requiere monitoreo, evaluación y actualización. Para Fischler (2012), la planificación también se puede entender como la administración colectiva del desarrollo urbano, ligado al uso

\footnotetext{
${ }^{2}$ En la Tabla 1, se puede ver que la legislación con mayor incidencia en un real proceso de descentralización está marcada en color gris. ${ }_{3}^{3}$ Para el Banco Mundial (2017), el concepto de gobernanza es el proceso por el cual el Estado y actores no pertenecientes al Estado, interactúan para diseñar e implementar políticas dentro de un conjunto de reglas formales e informales que dan forma y son formadas por el poder (Banco Mundial, 2017).

Para la ONU-Habitat III (2015), la gobernanza urbana es el software que permite que el hardware urbano pueda funcionar, un ambiente que requiere de marcos legales adecuados, eficiencia política, procesos administrativos y de gestión, como también instituciones locales fuertes y capaces de responder a las necesidades de los ciudadanos (ONU-Habitat III, 2015).
} 
de la deliberación intencional para dar forma a los asentamientos humanos. Es la mobilización de la voluntad de los diversos actores y agentes (incluyendo la comunidad), y el diseño de estrategias para crear, mejorar o preservar el entorno en que vivimos; este entorno es tanto físico (natural y construído), como cultural (social, económico y político) (Fischler, 2012).

Según la APA, el objetivo de la planificación yace en maximizar la salud, seguridad y bienestar económico de todas las personas que viven en nuestras comunidades; esto involucra pensar en cómo nos movemos en las zonas urbanas, como podemos atraer el desarrollo económico, dónde queremos vivir y qué oportunidades de espacios públicos queremos priorizar (APA, 2019). Para Lira (1990), planificar es más que un mero problema técnico del estado; es una actividad continua de respaldo a un arduo y lento proceso de diálogo y entendimiento social, protagonizado directamente por los actores reales, públicos y privados, como también por la difusa y creciente gama de las entidades no gubernamentales (Lira, 1990). Para efectos de este artículo se define el concepto como la planificación de todos los sistemas que permiten la existencia, el desarrollo y el funcionamiento de los asentamientos humanos, tanto a nivel urbano como a nivel regional; es la organización del desarrollo en el territorio e incluye una serie de políticas, planes y programas en el caso chileno.

Adicionalmente, resulta importante la discusión sobre cómo en el contexto de una gobernanza de características jerárquicas y verticales, una mayor estructura horizontal entre los niveles de planificación puede aportar a revertir la fragmentación y disociación del desarrollo local, regional y sectorial; para ello resulta clave examinar cómo se puede institucionalizar los nuevos espacios de interacción, como lo definen Elinbaum y Galland (2016), a modo de formas fluidas de planificación, para llegar a ofrecer soluciones más representativas y diversas.

\section{Metodología}

La metodología empleada en esta investigación es de carácter aplicado (Garza, 1996), empleando metodologías mixtas (cuantitativas y cualitativas). Se realizó una encuesta a nivel nacional (aplicada a todas las comunas del país), para el levantamiento de datos con respecto a las problemáticas planteadas. También se realizaron entrevistas semi-estructuradas a autoridades, profesionales y funcionarios de los gobiernos regionales respectivos y municipalidades, a modo de profundización del levantamiento de datos a partir de la encuesta señalada.

\section{Encuesta nacional y entrevistas específicas}

Se trabajó metodológicamente con una encuesta nacional a los asesores(as) urbanistas y/o a los(as) directores(as) de la Secretaría Comunal de Planificación (SECPLAN) de todas las comunas del país, logrando un total de 110 respuestas. A partir de los resultados de la encuesta, se analizaron los resultados de manera cuantitativa y descriptiva, tanto de manera agregada como también contrastando los resultados según las siguientes características:

o Si la comuna pertenece o no a la Región Metropolitana (RM).

o Si la comuna pertenece o no a un área metropolitana.

- Si la comuna se encuentra a una distancia mayor a 75 kilómetros con respecto a la capital regional.

o Si la comuna concentra una población menor a 70.000 habitantes, una población entre 70.000 y 150.000 hab., o una población mayor a 150.000 hab.

o Si el ingreso del municipio proveniente del Fondo Común Municipal (FCM) es superior a 50\% del ingreso municipal total.

Las características mencionadas se definen para contrastar cómo responden las diferentes comunas a las preguntas de la encuesta y si estas características son condicionantes para detectar distintos niveles de 
incidencia por parte de municipios en las decisiones del gobierno central con respecto a proyectos de transformación urbana. Tras el análisis de las respuestas de la encuesta se realizó una categorización de las comunas, para definir las entrevistas semi-estructuradas, de modo de profundizar en el análisis (Tabla 2).

\section{Entrevistas semi-estructuradas}

Se realizaron entrevistas semi-estructuradas a asesores urbanistas, directores y profesionales de la Secretaría de Planificación (SECPLA) de seis municipalidades del país, de modo de conocer cuál ha sido la experiencia, cuáles han sido las dificultades, cómo el municipio incide en y aborda la ejecución de un proyecto sectorial en su comuna. Se definieron seis tipos de comunas a partir de las tres características que tenían mayores diferencias en la forma de responder en la encuesta nacional.

\begin{tabular}{ccc}
\hline Pertenece a: & Población alta & Población baja \\
\hline Región Metropolitana & La Pintana | 177.335 hab. & Vitacura | 85.384 hab. \\
\hline Área Metropolitana & Concepción | 223.574 hab. & Penco | 47.367 hab. \\
\hline Ni RM/ ni AM & Punta Arenas | 131.592 hab. & Longaví | 30.534 hab. \\
\hline
\end{tabular}

Tabla 2. Tipologías de comunas para realización de entrevistas.

Fuente: Elaboración propia

De esta manera, se eligieron primero tres comunas con población alta y tres con población baja; de cada grupo de tres, una perteneciente a la Región Metropolitana, una perteneciente a un área metropolitana y una no perteneciente a áreas metropolitanas. Cabe señalar que para cada categoría se partió con un grupo más amplio de posibilidades y dependiendo de la respuesta por parte de la municipalidad (en algunos casos no hubo respuesta), se eligieron los siguientes casos. A continuación, se especifican las comunas elegidas:

\section{Resultados}

\section{Resultados encuesta nacional a municipalidades}

Los resultados obtenidos tanto a partir de la realización de la encuesta a municipalidades, y a partir del análisis tanto en forma agregada (Tabla 3), como por contraste (Tabla 5), dan cuenta de como los municipios inciden (o no inciden) en la acción planificadora proveniente del nivel de gobierno central. Se plantea que la incidencia puede tener tres niveles: (1) incidencia en la formulación del proyecto; (2) incidencia en el diseño del proyecto; (3) incidencia en la gestión del proyecto. Revisando los resultados agregados, se puede ver que las principales prioridades de las comunas encuestadas son los espacios públicos, verdes y plazas de la comuna. A su vez, la autoridad que tiene mayor incidencia en las decisiones vinculadas a temas de planificación es el(la) alcalde(sa), seguido por el(la) secretario(a) comunal de planificación (SECPLA). Cuando se anuncia un proyecto sectorial, la mayoría de las comunas (46.3\%) analizan posibles impactos que pueda haber en la comuna. A su vez, un 33.3\% toma rápidamente contacto con la contraparte. Con respecto a la pregunta sobre cuando un proyecto está en ejecución, un $47.6 \%$ de los municipios plantea que existe amplio contacto y trabajo en conjunto con la contraparte, pero a su vez, un $31.4 \%$ plantea que existe poco contacto con la contraparte. 


\begin{tabular}{l} 
Pregunta \\
\hline $2 \begin{array}{l}\text { En nuestra comuna, los temas de planificación que } \\
\text { tienen mayor prioridad son los siguientes. }\end{array}$
\end{tabular}

Alternativas seleccionadas con mayor frecuencia

Espacios públicos, espacios verdes y plazas

Vialidad, obras públicas y urbanización

En su municipio, ¿quién(es) deciden sobre los temas

3 de planificación territorial y localización de proyectos urbanos?

Alcalde

Secretario comunal de planificación (Secpla)

Analizamos posibles impactos que pueda haber en nuestra comuna.

4 Cuando se anuncia un proyecto sectorial...

Tomamos contacto con la contraparte rápidamente.

5 Cuando nos informamos sobre un proyecto sectorial...

Por lo general planteamos reparos tras revisar nuestros IPT.

Revisamos como el proyecto se ajusta a nuestros IPT.

6 Cuando el proyecto sectorial está en ejecución, Tenemos amplio contacto y realizamos nosotros... trabajo en conjunto con la contraparte.

Existe poco contacto con la contraparte.

7 Con los proyectos sectoriales que se realizan en nuestra comuna...

Mayormente tenemos éxito en sugerir cambios en los proyectos.

Rara vez tenemos éxito en sugerir cambios en los proyectos.

En general, el centralismo influye

8 ¿Cómo incide el centralismo, en la capacidad que negativamente en los resultados de los tienen de incidir en los proyectos sectoriales? proyectos.

No influye ya que las SEREMIs tienen un rol predominante.

Ni positivo ni negativo, depende del nivel

9 ¿Cómo calificaría el impacto de proyectos sectoriales en la planificación territorial que realizan a nivel local? de diálogo con la contraparte.

Positivo, refuerza las orientaciones y directrices de nuestros IPT.

Tabla 3. Incidencia local en proyectos sectoriales (resultados agregados encuesta nacional)

Fuente: Elaboración propia

Con respecto al planteamiento de observaciones y cambios a los proyectos, un $44.7 \%$ de las municipalidades tiene éxito en sugerir cambios en los proyectos sectoriales, y un 34.0\% rara vez tiene éxito en sugerir cambios. Con respecto a la incidencia del centralismo característico del país, en la capacidad de los municipios por influir en los resultados de los proyectos, un $58.4 \%$ plantea que el centralismo influye negativamente en los resultados de los proyectos, y un $21.8 \%$ no influye porque las SEREMIs asumen un rol importante y con ellos existe mayor trabajo en conjunto. Finalmente, cuando se preguntó sobre el impacto de los proyectos sectoriales en la calidad de vida de la comuna, un $47.4 \%$ respondió que no era positivo ni negativo y que dependía del nivel de diálogo con la contraparte. A su vez, un $23.7 \%$ de los municipios respondió que el impacto era positivo, que refuerza las orientaciones y directrices de los IPT. 
Al analizar los resultados de la encuesta aplicada características específicas ${ }^{4}$, y al elegir las preguntas que mejor ilustran la incidencia de los gobiernos locales en las decisiones de planificación provenientes del nivel central (preguntas 5, 6 y 8), las alternativas que tuvieron mayor frecuencia de respuesta fueron las siguientes (Tabla 4):

\begin{tabular}{cccc}
\hline Pregunta & $\begin{array}{c}\text { P5. Contacto con el } \\
\text { nivel de gobierno } \\
\text { central }\end{array}$ & $\begin{array}{c}\text { P6. Nivel de } \\
\text { receptividad }\end{array}$ & $\begin{array}{c}\text { P8. Impacto } \\
\text { proyectos nivel } \\
\text { central en local }\end{array}$ \\
\hline Alternativa & $\begin{array}{c}\text { (a) Como municipios } \\
\text { tenemos amplio } \\
\text { contacto y trabajo en } \\
\text { conjunto con el } \\
\text { gobierno central. }\end{array}$ & $\begin{array}{c}\text { (b) Como municipio } \\
\text { mayormente tenemos } \\
\text { éxito en sugerir } \\
\text { cambios a los } \\
\text { proyectos sectoriales. }\end{array}$ & $\begin{array}{c}\text { (c) Como municipio } \\
\text { pensamos que los } \\
\text { proyectos sectoriales } \\
\text { tienen un impacto ni } \\
\text { positivo ni negativo en } \\
\text { la planificación local, } \\
\text { depende del nivel de } \\
\text { diálogo. }\end{array}$ \\
\hline $\begin{array}{c}\text { Tasa de } \\
\text { respuesta }\end{array}$ & & & \\
$\begin{array}{c}\text { (\% municipios } \\
\text { que seleccionó } \\
\text { la alternativa) }\end{array}$ & $47.6 \%$ & $44.7 \%$ & $28.4 \%$ \\
\hline
\end{tabular}

Tabla 4. Preguntas sobre incidencia del nivel local y alternativas con mayor tasa de respuesta

Fuente: Elaboración propia

A su vez, en la Tabla 5 se puede notar que con respecto al contacto de las municipalidades con el nivel central en relación con proyectos sectoriales (P5), las mayores diferencias se dan cuando se contrastan las comunas con dependencia del FCM sobre 50\% con comunas con dependencia bajo $50 \%$, donde se genera una diferencia de 19.2 puntos para la pregunta 5, alternativa a, que trata sobre el contacto con el nivel central y como los municipios tienen amplio contacto y trabajo con el gobierno central. Los resultados muestran que las comunas con alta dependencia del FCM, el trabajo colaborativo es mayor que en las comunas con bajo nivel de dependencia del FCM.

Luego, cuando se contrasta comunas según su ubicación con respecto a la capital regional (comunas de distancia mayor a $75 \mathrm{~km}$. con comunas ubicadas a menos de $75 \mathrm{~km}$.), se genera una diferencia de 14.2 puntos para la pregunta 5, alternativa a, que al igual que en el caso anterior trata sobre el contacto con el nivel central y como los municipios tienen amplio contacto y trabajo con el gobierno central. Los resultados muestran que las municipalidades ubicadas a menos de $75 \mathrm{kms}$. de la capital regional, tienen mayor contacto y trabajo

\footnotetext{
${ }^{4}$ Las características son las siguientes: si la comuna pertenece o no a la región metropolitana (RM); si la comuna pertenece o no a un área metropolitana; si la comuna se encuentra a una distancia mayor a 75 kilómetros con respecto a la capital regional; si la comuna concentra una población menor a 70.000 habitantes, una población entre 70.000 y 150.000 hab., o una población mayor a 150.000 hab; si el ingreso del municipio proveniente del Fondo Común Municipal (FCM) es superior a 50\% del ingreso municipal total.
} 
colaborativo con el nivel central (50.0) en comparación con aquellas ubicadas a una distancia superior a 75 kms. de la capital regional (35.8).

También es significativa la diferencia que se genera cuando se comparan comunas según su población, para la pregunta 5, alternativa a. En las municipalidades con mayor población, hay mayor contacto colaborativo con el nivel central, versus aquellas con menor población, generando una diferencia de 10 puntos. Con respecto al nivel de receptividad que tiene el nivel central para recibir las sugerencias de los municipios a los proyectos sectoriales (P6), la única diferencia significativa se da cuando se compara según comunas pertenecientes a la región metropolitana con aquellas no pertenecientes a la región metropolitana, generando una diferencia de 19.3 puntos, en que las comunas no-RM tienen mayor éxito en sugerir cambios a proyectos sectoriales que las comunas RM.

\begin{tabular}{|c|c|c|c|c|c|c|c|c|c|c|c|c|c|c|c|}
\hline $\begin{array}{c}\text { Pregunt } \\
\quad a\end{array}$ & $\begin{array}{l}R M \\
-s i\end{array}$ & $\begin{array}{l}R M \\
-n o\end{array}$ & dif. & $\begin{array}{l}A M \\
-s i\end{array}$ & $\begin{array}{l}A M \\
-n o\end{array}$ & dif. & $\begin{array}{l}C+ \\
75\end{array}$ & $\begin{array}{l}C- \\
75\end{array}$ & dif. & $\begin{array}{c}\text { pob } \\
>15 \\
0\end{array}$ & $\begin{array}{l}\text { pob } \\
<70\end{array}$ & dif. & $\begin{array}{c}F C \\
M> \\
50\end{array}$ & $\begin{array}{c}F C \\
M< \\
50\end{array}$ & dif. \\
\hline P5 & $\begin{array}{c}44 . \\
4\end{array}$ & $\begin{array}{c}42 . \\
9\end{array}$ & 1.5 & $\begin{array}{c}46 . \\
7\end{array}$ & 42 & 4.7 & $\begin{array}{c}35 . \\
8\end{array}$ & 50 & $\begin{array}{c}14 . \\
2\end{array}$ & 60 & 50 & 10 & $\begin{array}{c}53 . \\
1\end{array}$ & $\begin{array}{c}33 . \\
9\end{array}$ & $\begin{array}{c}19 . \\
2\end{array}$ \\
\hline P6 & $\begin{array}{c}25 . \\
9\end{array}$ & $\begin{array}{c}45 . \\
2\end{array}$ & $\begin{array}{c}19 . \\
3\end{array}$ & $\begin{array}{c}36 . \\
7\end{array}$ & 42 & 5.3 & $\begin{array}{c}41 . \\
5\end{array}$ & $\begin{array}{c}39 . \\
7\end{array}$ & 1.8 & 40 & $\begin{array}{c}41 . \\
6\end{array}$ & 1.6 & $\begin{array}{c}40 . \\
8\end{array}$ & $\begin{array}{c}40 . \\
3\end{array}$ & 0.5 \\
\hline P8 & $\begin{array}{c}48 . \\
1\end{array}$ & $\begin{array}{c}38 . \\
1\end{array}$ & 10 & $\begin{array}{c}46 . \\
7\end{array}$ & $\begin{array}{c}38 . \\
3\end{array}$ & 8.4 & $\begin{array}{c}39 . \\
6\end{array}$ & $\begin{array}{c}41 . \\
4\end{array}$ & 1.8 & 46 & $\begin{array}{c}41 . \\
4\end{array}$ & 4.6 & $\begin{array}{c}40 . \\
8\end{array}$ & $\begin{array}{c}40 . \\
3\end{array}$ & 0.5 \\
\hline
\end{tabular}

Tabla 5. Incidencia del nivel local (diferenciación por características)

Fuente: Elaboración propia

Finalmente, en relación con el impacto de los proyectos sectoriales en la planificación local (P8), la única diferencia significativa en la forma de responder se genera cuando se contrastan comunas pertenecientes a la región metropolitana con aquellas no pertenecientes, generando una diferencia de 10 puntos para la alternativa $c$ sobre el impacto de proyectos sectoriales en el nivel local. Los resultados muestran que las comunas RM responden con mayor frecuencia que el impacto será positivo o negativo dependiendo del nivel de diálogo, comparado con las comunas no-RM.

\section{Resultados entrevistas semi-estructuradas}

Con las entrevistas realizadas según las tipologías definidas (Tabla 2), se buscó complementar los datos obtenidos mediante la encuesta. Primero se hizo un catastro de los grandes proyectos urbanos de los últimos 20 años (Tabla 6), y en base a éstos se buscó explorar cuáles eran los factores que favorecieron y dificultaron la capacidad de la municipalidad de incidir en decisiones del nivel central. Tras el análisis y síntesis de los datos obtenidos a partir de las entrevistas, se pueden apreciar experiencias disímiles con respecto a la capacidad de las municipalidades seleccionadas para incidir en las decisiones de proyectos de inversión provenientes desde el nivel central mediante los ministerios sectoriales o desde el nivel del gobierno regional mediante el Fondo Nacional de Desarrollo Regional (FNDR). 


\begin{tabular}{|c|c|c|}
\hline La Pintana & Concepción & Punta Arenas \\
\hline $\begin{array}{l}\text { Plan del gobierno regional de } \\
\text { intervenir la población El } \\
\text { Castillo; proyecto de } \\
\text { regeneración urbana sector } \\
\text { Santo Tomás; proyecto de } \\
\text { nueva vivienda social sector } \\
\text { Antumapu; proyecto para } \\
\text { desarrollar el PRC y Pladeco } \\
\text { comunal; modificación PRMS; } \\
\text { corredor Santa Rosa; proyecto } \\
\text { acceso sur de Santiago. }\end{array}$ & $\begin{array}{l}\text { Mejoramiento de accesibilidad } \\
\text { y conexión vial de Concepción; } \\
\text { camino a Cabrero; centros } \\
\text { comerciales de gran tamaño; } \\
\text { paseo peatonal Eje } \\
\text { Bicentenario; Corredor } 21 \text { de } \\
\text { mayo; Parque Metropolitano } \\
\text { Cerro Caracol; canalización } \\
\text { Río Andalien; circuito } \\
\text { intercomunal de ciclovías; } \\
\text { proyectos de accesibilidad } \\
\text { universal y pavimentación; } \\
\text { Laguna Redonda. }\end{array}$ & $\begin{array}{l}\text { Apertura de la costanera hacia } \\
\text { el estrecho (de Magallanes); } \\
\text { desarrollo de infraestructura } \\
\text { comercial (mall, casino, } \\
\text { tiendas); construcción del } \\
\text { hospital regional (se terminó de } \\
\text { construir el año 2012). }\end{array}$ \\
\hline Vitacura & Penco & Longaví \\
\hline $\begin{array}{l}\text { Autopista Costanera Norte; } \\
\text { Parque Bicentenario; apertura } \\
\text { Constanera Sur; consolidación } \\
\text { circuito 42K; proyecto vial } \\
\text { Santiago centro-oriente; } \\
\text { licitación MOP AVO subsuelo. }\end{array}$ & $\begin{array}{l}\text { Bypass Penco; mejoramiento } \\
\text { de la plaza Los } \\
\text { Conquistadores; proyecto de } \\
\text { renovación de luminarias; } \\
\text { mejoramiento ruta } 150 \\
\text { Concepción-Penco; puesta en } \\
\text { valor del fuerte La Planchada. }\end{array}$ & $\begin{array}{l}\text { Remodelación de la plaza de } \\
\text { armas; mejoramiento vialidad; } \\
\text { proyectos de vivienda social. }\end{array}$ \\
\hline
\end{tabular}

Tabla 6. Grandes proyectos urbanos y transformaciones

Fuente: Elaboración propia

En las siguientes Tablas ( 7 y 8), se pueden visualizar una síntesis de los resultados. Por su tamaño, el caso de la municipalidad de Concepción no solo cuenta con una cantidad mayor de transformaciones urbanas, sino que cuenta con mayor cantidad de factores influyentes en una mayor capacidad de incidir en decisiones del nivel central. Entre estas herramientas está: (1) la existencia del Directorio Urbano de Concepción; (2) buenas relaciones de trabajo y en equipo con el gobierno regional y las SEREMIs, que se debe a un liderazgo basado en respecto; (3) presencia de una cultura institucional que piensa en la ciudad a largo plazo, más que en beneficios políticos de corto plazo; (4) un involucramiento activo de la ciudadanía y de otros actores y organizaciones en la elaboración de IPT (Ej. caso del PLADECO participativo). Entre los factores que dificultaban la capacidad de incidir, se mencionó que la distancia con respecto a Santiago dificultaba la capacidad de conseguir recursos. Por otra parte, el caso de la municipalidad de Penco, también cuenta con varios factores que favorecen la incidencia. Al pertenecer también al área metropolitana de Concepción, son invitados al Directorio Urbano de Concepción, lo que favorece la incidencia en torno a proyectos provenientes del nivel regional y central. En cuanto a las dificultades, comentan que como no es obligatoria la participación resolutiva de la municipalidad en proyectos sectoriales, aún no es mucha la participación en ese sentido. 


\begin{tabular}{|c|c|c|}
\hline La Pintana & Concepción & Punta Arenas \\
\hline $\begin{array}{l}\text { La llegada de un nuevo equipo } \\
\text { profesional (tras la llegada de } \\
\text { un nuevo alcalde), habría } \\
\text { generado en el último año una } \\
\text { ruptura con resultados positivos } \\
\text { con respecto a cómo se venían } \\
\text { manejando las decisiones y los } \\
\text { planes de planificación } \\
\text { territorial. }\end{array}$ & $\begin{array}{l}\text { Presencia del Directorio } \\
\text { Urbano; capacidad de trabajo } \\
\text { en equipo entre la } \\
\text { municipalidad, GORE y } \\
\text { SEREMIs; estilo de liderazgo } \\
\text { basado en el respeto; } \\
\text { presencia de una cultura } \\
\text { institucional que piensa en la } \\
\text { ciudad más que en los } \\
\text { beneficios políticos; un enfoque } \\
\text { puesto en mejorar la ciudad; } \\
\text { involucramiento activo de la } \\
\text { ciudadanía y de actores en la } \\
\text { elaboración de IPT (Pladeco); } \\
\text { equipo profesional municipal } \\
\text { fortalecido. }\end{array}$ & $\begin{array}{l}\text { El caso del plan de zonas } \\
\text { extremas de los gobiernos } \\
\text { regionales fue considerado por } \\
\text { los profesionales del municipio } \\
\text { como un acto democrático y } \\
\text { descentralizado, ya que los } \\
\text { municipios pudieron priorizar } \\
\text { los proyectos según su } \\
\text { urgencia y VAN social no era } \\
\text { requisito. }\end{array}$ \\
\hline Vitacura & Penco & Longaví \\
\hline $\begin{array}{l}\text { El entrevistado no hizo } \\
\text { referencia a factores que } \\
\text { favorecen la capacidad } \\
\text { municipal de incidir. }\end{array}$ & $\begin{array}{l}\text { Cuando se trata de proyectos } \\
\text { FNDR a cargo del gobierno } \\
\text { regional, hay una mayor } \\
\text { posibilidad de participación por } \\
\text { parte de la municipalidad; } \\
\text { invitación por parte de la } \\
\text { municipalidad de Concepciónal } \\
\text { Directorio Urbano para discutir } \\
\text { proyectos de escala } \\
\text { metropolitana. }\end{array}$ & $\begin{array}{l}\text { El entrevistado no hizo } \\
\text { referencia a factores que } \\
\text { favorecen la capacidad } \\
\text { municipal de incidir. }\end{array}$ \\
\hline
\end{tabular}

Tabla 7. Factores que favorecen la capacidad de la municipalidad para incidir en proyectos sectoriales

Fuente: Elaboración propia

En el caso de Punta Arenas, se aprecia un menor nivel de proyectos y un nivel bajo de incidencia en torno a las decisiones, planificación y gestión en relación con éstos. Se mencionó el caso del Plan de Financiamiento de Zonas Extremas de GORE, como un programa de inversión importante hasta el año 2020, que tomó en consideración la opinión de la municipalidad, ya que ellos pudieron definir el orden de prioridades a partir de una cartera de proyectos específica. El caso de La Pintana es particular ya que si bien se trata de una comuna que históricamente cuenta con escasos recursos y se encuentra cargada de problemáticas sociales, en el último año cuenta con un equipo profesional renovado (después de más de veinte años), con una fuerte motivación que busca avanzar con proyectos omo la elaboración de un PRC y un nuevo PLADECO, como también por dialogar con el nivel sectorial para asegurar que no se vuelvan a repetir errores pasados de políticas públicas fallidas. 


\begin{tabular}{|c|c|c|}
\hline La Pintana & Concepción & Punta Arenas \\
\hline $\begin{array}{l}\text { El hecho de que La Pintana ha } \\
\text { sido históricamente afectada } \\
\text { por políticas públicas fallidas. }\end{array}$ & $\begin{array}{l}\text { Distancia con respecto a } \\
\text { Santiago dificulta la capacidad } \\
\text { de conseguir los recursos para } \\
\text { algunos proyectos. }\end{array}$ & $\begin{array}{l}\text { Falta de involucramiento y } \\
\text { retroalimentación por parte de } \\
\text { los ministerios con respecto a } \\
\text { proyectos, lo que lleva a } \\
\text { decisiones mal tomadas; como } \\
\text { son escasos los proyectos de } \\
\text { inversión pública la } \\
\text { municipalidad tiende a no poner } \\
\text { problema, de modo que se } \\
\text { concrete el proyecto. }\end{array}$ \\
\hline Vitacura & Penco & Longaví \\
\hline $\begin{array}{l}\text { El entrevistado no hizo } \\
\text { referencia a factores que } \\
\text { dificultan la capacidad } \\
\text { municipal de incidir. }\end{array}$ & $\begin{array}{l}\text { El hecho de que no es } \\
\text { obligatoria una participación } \\
\text { resolutiva por parte de la } \\
\text { municipalidad en las decisiones } \\
\text { con respecto a proyectos } \\
\text { sectoriales; el hecho de que el } \\
\text { territorio comunal de Penco en } \\
\text { realidad funciona como un } \\
\text { lugar de paso en el proceso de } \\
\text { desarrollo de infraestructura } \\
\text { vial relacionada a la industria } \\
\text { de la región. }\end{array}$ & $\begin{array}{l}\text { Falta de involucramiento y } \\
\text { retroalimentación por parte de } \\
\text { los ministerios con respecto a } \\
\text { proyectos, lo que lleva a } \\
\text { decisiones mal tomadas. }\end{array}$ \\
\hline
\end{tabular}

Tabla 8. Factores que dificultan la capacidad de la municipalidad para incidir en proyectos sectoriales

Fuente: Elaboración propia

En relación con el caso de Longaví, según lo conversado con el entrevistado, se vislumbra baja participación e incidencia en la planificación territorial proveniente del nivel central, debido a una falta de retroalimentación por parte de los ministerios cuando desde la municipalidad se hacen observaciones. La municipalidad sí se preocupa de los temas pero ven que hay escasas oportunidades de involucramiento. Sí cumplen con un rol importante en cuanto a recibir solicitudes de vivienda de los habitantes de la comuna y hacerlas saber al ministerio respectivo. Finalmente, para el caso de Vitacura, el acceso a información sobre las fortalezas y dificultades de la municipalidad para incidir en la planificación proveniente del nivel central fue más limitado que en los otros casos y no se lograron respuestas específicas a estos puntos.

\section{Conclusiones y reflexiones finales}

En este artículo se ha revisado desde una perspectiva crítica, los principales hitos legislativos que han estructurado los procesos de descentralización de la planificación territorial en Chile, desde el año 1925 al 2018. A partir del reconocimiento de un proceso tardío y fragmentado de los procesos de descentralización (Tabla 1), evidenciando un proceso dicotómico entre el desarrollo de los procesos legislativos de descentralización político-administrativa, y aquellos de regulación del desarrollo urbano y planificación territorial, se ha querido analizar cuáles son los efectos en la planificación territorial que se realiza desde los distintos niveles de gobierno. Se ha buscado analizar en particular, cómo son las dinámicas entre el nivel central 
y las municipalidades en Chile, desde la perspectiva de la capacidad de las últimas por incidir en la formulación, diseño y ejecución de proyectos de inversión sectorial.

A partir de la realización de la encuesta, se puede ver que la mayoría de las comunas encuestadas toma un rol activo frente al anuncio de un proyecto de inversión sectorial (79.6\%), ya sea analizando posibles impactos como rápidamente haciendo contacto con la contraparte. Otro resultado hallazgo interesante a partir de la encuesta, muestra como cuando un proyecto sectorial está en ejecución, un $47 \%$ de los municipios plantea que existe amplio contacto con el nivel central, versus un $31.4 \%$ que plantea que existe poco contacto con la contraparte. También se encontró que un $44.7 \%$ de las municipalidades tiene éxito en sugerir cambios a los proyectos, versus un $34 \%$ que no tuvo el mismo éxito. Por último, otro hallazgo significativo fue que un $47.4 \%$ de las municipalidades respondió que el impacto de los proyectos sectoriales no era positivo ni negativo, sino que depende de el nivel de diálogo con la contraparte del nivel central. Estos resultados reflejan que existe un interés desde el nivel local por incidir y participar en la formulación, diseño y ejecución de los proyectos sectoriales, pero que no siempre se genera un diálogo con la contraparte del nivel central (ya sea en el ministerio como con las SEREMIs), y que esa interacción es clave para lograr resultados que mejoren la calidad de vida en los territorios.

También a partir de la encuesta nacional, se pudo ver como ciertas características o factores influyen en el nivel de incidencia de los gobiernos locales en los proyectos sectoriales. En ese sentido, resulta curioso como en comunas con alta dependencia al Fondo Común Municipal (FCM), el trabajo colaborativo con el nivel central es mayor que para el caso de comunas con bajo nivel de dependencia al FCM. Como existe una alta correlación entre dependencia al FCM y bajos ingresos totales municipales, este resultado habla que las municipalidades con menores ingresos tienden a tener más instancias de trabajo colaborativo en relación con proyectos sectoriales. Una hipótesis en relación con ese resultado es que desde el nivel central hay un cuidado especial por lograr un trabajo colaborativo en municipalidades más vulnerables donde probablemente hay una menor cantidad total de inversión (pública y privada).

A su vez, se encontró que las municipalidades ubicadas a menos de $75 \mathrm{kms}$. de la capital regional tienen mayor contacto y trabajo colaborativo con el nivel central, en comparación con aquellas comunas ubicadas a una distancia superior a $75 \mathrm{kms}$. de la capital regional. Este indicador puede ser reflejo de cómo la instancia de coordinación con las SEREMIs en la capital regional es importante, y que una mayor distancia física sigue siendo un factor relevante a la hora de realizar trabajo de coordinación en torno a proyectos sectoriales. Por último, también resulta interesante como la encuesta arroja resultados que muestran que, en las comunas no pertenecientes a la Región Metropolitana, hay mayor éxito en sugerir cambios a proyectos sectoriales en comparación con las comunas pertenecientes a la Región Metropolitana. Una hipótesis en relación con este resultado es que, en la Región Metropolitana, los ministerios que históricamente han estado basados en la capital nacional pueden ejercer una mayor injerencia, relacionada a un sentido de pertinencia territorialinstitucional, lo que lleva al ejercicio de un mayor dominio por las decisiones vinculadas a los proyectos.

A partir de una profundización en seis casos, incluyendo las municipalidades de La Pintana, Vitacura, Concepción, Penco, Punta Arenas y Longaví, se pudo levantar información sobre los principales proyectos y transformaciones urbanas de los últimos 20 años, y sobre los factores que facilitaron o dificultaron una mayor incidencia por parte de la municipalidad en los proyectos de inversión sectorial. Entre las capacidades que facilitan el trabajo colaborativo se encuentran factores como la renovación de equipos profesionales y técnicos en la municipalidad, la presencia de instancias de diálogo entre los niveles de gobierno y los sectores público y privado, el desarrollo de proyectos a nivel regional (financiamiento FNDR) versus desde el nivel central (financiamiento sectorial). Entre los factores que dificultan el trabajo colaborativo, destaca el tema de la distancia con respecto a Santiago, el hecho de que aún no es obligatoria la participación resolutiva de las municipalidades en proyectos sectoriales, la falta de retroalimentación por parte del nivel central con respectos a los proyectos en desarrollo, y la escasez de recursos de inversión en proyectos de carácter urbano destinados 
desde el nivel central a ciertas comunas, lo que genera una situación en que la municipalidad no quiere plantear observaciones por temor a perder el proyecto.

En los resultados se destacó el caso de Concepción, por la forma en que han desarrollado capacidad de incidencia basada en un trabajo colaborativo en una mesa que reúne autoridades y representantes de la municipalidad, las SEREMIs, el gobierno regional, actores privados y académicos (Directorio Urbano). Los entrevistados plantean que la instancia de diálogo que se da en la mesa ha sido clave para desarrollar un mayor respecto en torno a opiniones, y una "cultura de ciudad" que busca pensar más allá que la agenda política del momento y plantear proyectos de largo plazo en pos de mejorar la calidad de vida de la ciudadanía.

De esta manera, el caso de Concepción—en que se generaron espacios de diálogo, coordinación y cooperación entre los niveles de gobierno, el sector privado y la academia—se aproxima a las definiciones de planificación expuestas en el marco teórico de este artículo, en que se plantea la importancia de avanzar hacia un arduo proceso de diálogo y entendimiento social, protagonizado por diferentes actores públicos y privados. También se aproxima como ejemplo a las ideas en torno a los beneficios de una gobernanza de estructura horizontal, de modo de revertir la fragmentación y disociación entre los niveles de gobierno. Inclusive, es un ejemplo de que se ha avanzado a institucionalizar los espacios de interacción (Directorio Urbano de Concepción), propiciando las formas fluidas de planificación que mencionan Elinbaum y Galland (2016), a pesar de la presencia de un gobierno altamente centralizado. Para futuras discusiones, se pueden explorar hipótesis que permitan entender de mejor manera por qué se generan nuevas formas fluidas de planificación en algunas zonas urbanas del país y no en otras, y cuáles son las condiciones necesarias para que se produzcan y consolide una nueva institucionalidad horizontal y flexible entre la institucionalidad tradicional existente.

\section{BIBLIOGRAFÍA}

AMERICAN PLANNING ASSOCIATION APA, (2019). ¿What is planning? Información disponible en el vínculo: https://www.planning.org/aboutplanning/

BANCO MUNDIAL. (2017). World Development Report 2017. Governance and The Law. A World Bank Group Flagship Report.

BERKE, P., GODSCHALK, D., \& KAISER, E. (2006). Urban Land Use Planning (5th ed). Champaign, IL: University of Illinois Press.

BORJA, J. Y CASTELLS, M. (1998). Local y global: La gestión de las ciudades en la era de la información [Local and Global: The Development of Cities in the Information Era]. Madrid: Santillana.

BRENNER, N. (2003). La formación de la ciudad global y el re-escalamiento del espacio del Estado en la Europa Occidental post-fordista. EURE (Santiago) 29: 05-35.

BRENNER, N. (2004). Urban governance and the production of new state spaces in Western Europe, 1960-2000. Review of International Political Economy, 11 (3),447-488.

BRENNER, N. (2006). New State Spaces: Urban Governance and the Rescaling of Statehood. Oxford: Oxford University Press.

ELINBAUM, P. Y GALLAND, D. (2016). La redefinición de las escalas territoriales a través de la ordenación territorial, en Orellana, A. Link, y F. Noyola, J. Eds. (2016) Urbanización planetaria y la reconstrucción de la ciudad. Santiago: RIL editores - Instituto de Estudios Urbanos y Territoriales UC, 2016. 
FALLETI, T.G. (2010). Decentralization and subnational politics in Latin America, London: Cambridge: Cambridge University Press.

FINOT, I. (2001). Descentralización en América Latina: Teoría y práctica. Publicaciones CEPAL.

FISCHLER, R. (2012). Fifty theses on urban planning and urban planners. Journal of Planning Education and Research, 32, 1, 107-114.

GARZA, A. (1996). Manual de técnicas de la investigación para estudiantes de ciencias sociales. 6a edición, México: El Colegio de México, Biblioteca Daniel Cosío Villegas, 2004, 1996.

GROSS, P. (1991). Santiago de Chile (1925-1990): Planificación urbana y modelos políticos. EURE Revista Latinoamericana de Estudios Urbano Regionales, vol. 17, núm. 52-53, diciembre de 1991.

LIRA, L. (1990). La confección de Estrategias de Desarrollo Regional. Arenas, F. (ed.), Manual de desarrollo regional. Santiago: INC-PRED-REGION-IEU, 41-68.

MARSHALL, C. (2018). La dicotomía de los procesos de descentralización administrativa y planificación urbano-regional en Chile; efectos sobre la planificación y gestión subnacional. Tesis de doctorado. Facultad de Arquitectura, Diseño y Estudios Urbanos, Pontificia Universidad Católica de Chile.

NICKSON, A. (2003). Descentralización Política. En Blanes, J. y Pabón, E. (Comp.). (2003). La descentralización en América Latina, Logros y Desafíos. El papel de la Cooperación Europea.

OECD (2013), OECD Urban Policy Reviews, Chile 2013, OECD Publishing. http://dx.doi.org/10.1787/9789264191808-en

ONU-HABITAT III. (2015). Issue Papers - Urban Governance. Habitat III - United Nations Conference on Housing and Sustainable Urban Development. New York, 31 Mayo 2015

PALMA, E. Y RUFIÁN, D. (1989). Las nociones de centralización y descentralización. ILPES, Documento CPS48, Santiago de Chile.

RONDINELLI, D. (1981). Government Decentralization in Comparative Perspective: Theory and Practice in Developing Countries, International Review of Administrative Sciences, vol. 47, núm. 2.

RONDINELLI, D., NELLIS, J., AND CHEEMA, G. (1984). Decentralization in developing countries. A review of recent experience. Washington, DC: The World Bank.

RONDINELLI, D. ET AL. (1989). Analysing Decentralization Policies in Developing Countries: a PoliticalEconomy Framework. Development and Change, vol. 20, n. 1

TOCQUEVILLE, A. (1835) Democracy In America. New York :G. Dearborn \& Co. Print.

BANCO MUNDIAL. (2017). World Development Report 2017. Governance and The Law. A World Bank Group Flagship Report. 